\title{
Selective dorsal rhizotomy for spasticity not associated with cerebral palsy: reconsideration of surgical inclusion criteria
}

\author{
William C. Gump, M.D., ${ }^{1}$ Ian S. Mutchnick, M.D., M.S., ${ }^{1}$ \\ and Thomas M. Moriarty, M.D., Ph.D., \\ 'Division of Pediatric Neurosurgery, Norton Neuroscience Institute and Kosair Children's Hospital; and \\ ${ }^{2}$ Department of Neurological Surgery, University of Kentucky College of Medicine, Lexington, Kentucky
}

\begin{abstract}
Children with spastic diplegia from cerebral palsy (CP) experience measurable improvement in their spasticity and motor function following selective dorsal rhizotomy (SDR). The role of this operation in the treatment of other spasticity causes is less well defined. A literature review was undertaken to survey outcomes from SDRs performed outside the CP population. Multiple sclerosis was the most common diagnosis found, accounting for 74 of 145 patients described. Selective dorsal rhizotomies have also been reported in patients with traumatic brain and spinal cord injuries, ischemic and hemorrhagic stroke, neurodegenerative disease, hypoxic encephalopathy, and other causes of spasticity. Outcomes from surgery are generally described as favorable, although postoperative assessments and follow-up times are not standardized across reports. Long-term outcomes are sparsely reported. Larger numbers of patients and more detailed outcomes data have the potential to form a basis for expanding the inclusion criteria for SDR.
\end{abstract}

(http://thejns.org/doi/abs/10.3171/2013.8.FOCUS13294)

KeY WoRds
hypertonia $\quad$ selective dorsal rhizotomy $\quad \bullet \quad$ spasticity $\quad \bullet \quad$ cerebral palsy $\quad \bullet$

$\mathrm{S}$ ELECTIVE dorsal rhizotomy (SDR) is a standard treatment option for spastic paraparesis associated with cerebral palsy (CP) in selected patients. However, the diagnosis of CP encompasses a broad variety of underlying pathologies that appear early in life secondary to a brain lesion or dysfunction that is not the result of progressive or degenerative brain disease. ${ }^{2}$ Cerebral palsy arises from a diversity of causes and can affect widely variable anatomy. Despite this heterogeneity, outcomes and complications of SDR within this patient population have been extensively documented, and SDR is considered a safe and effective procedure. ${ }^{8,20,24,33}$ As this procedure and its utilization have matured, various inclusion and exclusion criteria for assessing a patient's candidacy for this surgery have been established.

Therapeutic rhizotomy for spasticity in adults has been performed on ventral nerve roots,,$^{22}$ but this approach was abandoned due to resultant muscle atrophy and loss of function. ${ }^{1}$ Laboratory work, however, had suggested that dorsal rhizotomy could be comparably effective in abolishing spasticity while avoiding similar adverse effects. ${ }^{30}$ This procedure was first described in children with cerebral spasticity who underwent complete dorsal rhizotomies of L2-3 and L5-S1, with results described as very good for resolution of spasticity but causing sig-

Abbreviations used in this paper: ALS = amyotrophic lateral sclerosis; $\mathrm{CP}=$ cerebral palsy; $\mathrm{MS}=$ multiple sclerosis; $\mathrm{SDR}=$ selective dorsal rhizotomy. nificant sensory loss and ataxia. ${ }^{11}$ The technique of isolating dorsal lumbar nerve fascicles and dividing only those demonstrating some type of abnormal physiological response on electrical stimulation was developed later., ${ }^{3,9,25}$ This strategy resulted in a significant reduction in adverse effects without loss of efficacy in spasticity relief, as verified in multiple randomized studies. ${ }^{8,20,21,33}$ Nonselective dorsal rhizotomy, in which some dorsal nerve rootlets (usually about 50\%) are divided without neurophysiological investigation, appears to produce similar results. ${ }^{27}$

Patient selection is important in maximizing chances for a satisfactory result from SDR. Although the specific criteria for SDR vary by institution, widely adopted guidelines have been published. ${ }^{24}$ Indications and contraindications for SDR as delineated by Park are summarized in Table 1. Patients with dystonia or athetoid movement disorders do not benefit from SDR. Most SDR studies have focused on children with $\mathrm{CP}^{;} ;, 20,21,33$ clinical conditions such as spastic hemiplegia, and diagnoses such as hypoxic encephalopathy as is noted in cases of near drowning, are generally listed as contraindications to SDR. ${ }^{24}$ Assessing the suitability of patients for SDR who fall outside traditional parameters is restricted by limited data.

\section{Methods}

A PubMed query was performed for English language articles published between January 1980 and June 
W. C. Gump, I. S. Mutchnick, and T. M. Moriarty

TABLE 1: Summary of published criteria for assessing suitability for SDR*

Indications
diagnosis of spastic diplegia or quadriplegia
age $2-45$ yrs
history of premature birth or neonatal asphyxia
bilateral schizencephaly underlying spastic diplegia
emerging locomotive functions
potential for significant postop functional gains
Contraindications
CP associated w/ intrauterine encephalitis
mixed CP w/ predominant dystonia, rigidity, athetosis, or ataxia
spastic hemiplegia
severe head injury \& hypoxic encephalopathy (near drowning)
familial spastic paraplegia \& other progressive neurological disorders
severe basal ganglia injury in children younger than 5 yrs
severe thoracolumbar scoliosis or lumbar lordosis
multiple prior muscle \& tendon releases
profound motor impairment w/ no head control
psychiatric disorder in adults
lack of commitment to carry out postop therapy

* According to Park, 2000.

2013 using the following search keywords in various combinations: selective, partial, dorsal rhizotomy, posterior rhizotomy, $\mathrm{CP}$, congenital brain malformation, spasticity. Papers describing SDR in patients without CP were selected. References from all selected papers were further examined for additional suitable studies.

\section{Results}

Utilization of SDR outside the context of CP-associated spastic paraparesis is sparsely documented, but these operations have been described as generally successful. These cases include patients with diagnoses of poststroke spasticity, ${ }^{14}$ neurodegenerative disease,${ }^{16}$ multiple sclerosis (MS), ${ }^{30}$ hydrocephalus, near drowning, and spinal cord pathology, including transverse myelitis and myelomeningocele. ${ }^{29}$ A summary of diagnoses represented in the available literature is illustrated in Table 2 .

\section{Case Series With Multiple Spasticity Causes}

A case series of 30 patients undergoing SDR between 1989 and 1991 included only 20 patients with a diagnosis of CP. ${ }^{29}$ Causes of spasticity in the other 10 patients were cerebral in 7 and spinal in 3 . These 10 patients included 2 patients each with hydrocephalus, near drowning, and hypoxia during heart surgery; and 1 patient each with head injury, transverse myelitis, MS in the spinal cord, and myelomeningocele. Among the 27 total patients with spasticity of cerebral origin, 19 had spastic quadriplegia preoperatively and 8 had spastic diplegia. Surgical results were not individually specified by origin, although most patients were described as achieving significant benefit.

Results were described as satisfactory in $100 \%$ of cas-
TABLE 2: Summary of non-CP associated causes of spasticity in reported patients who underwent SDR*

\begin{tabular}{lc}
\hline \multicolumn{1}{c}{ Diagnosis } & No. of Cases \\
\hline multiple sclerosis & 74 \\
spinal cord injury & 35 \\
neurodegenerative disease including ALS & 9 \\
traumatic brain injury & 8 \\
congenital brain malformation & 2 \\
hemorrhagic stroke & 2 \\
ischemic stroke & 2 \\
hydrocephalus & 2 \\
hypoxia during heart surgery & 2 \\
near drowning & 2 \\
transverse myelitis & 2 \\
brain tumor & 1 \\
hereditary spastic paraparesis & 1 \\
meningitis & 1 \\
myelomeningocele & 1 \\
myelopathy, unspecified & 1 \\
total & 145 \\
\hline
\end{tabular}

* Based on the following studies: 5,13-15,21,24,26,27,30,33,36.

es. Patients with spastic quadriplegia consistently showed significant improvement in activities of daily living, as well as some inconsistent improvement in swallowing, reduced irritability, and seizure control. Among the 8 patients with spastic diplegia, the 2 patients who were ambulatory both improved their gait pattern, 4 nonambulatory patients improved their sitting and standing skills, and 2 previously nonambulatory patients attained assisted gait within 1-2 years after surgery. The patient with myelomeningocele also improved from nonambulatory to assisted gait.

A larger and more recently published report described outcomes in 154 patients undergoing SDR for various spasticity causes at a single institution over the years 1969-1998. ${ }^{28}$ The indication for surgery was described as spasticity that interfered with daily living and was resistant to conservative therapy. The most common diagnosis in this cohort was CP, which accounted for 60 patients. There were 52 patients with MS, 30 with spine trauma, 5 with head trauma, 4 with spinocerebellar degenerative disease, and 3 with amyotrophic lateral sclerosis (ALS). No other reports of SDR for ALS-associated spasticity were identified.

In this study, spasticity outcomes were measured with the Modified Ashworth Scale and assessed preoperatively, early (less than 1 year after surgery), and late (more than 1 year postoperatively). Patients with MS had a mean improvement on the Modified Ashworth Scale of 3 points early and 2 points late when compared with preoperative baseline. A similar pattern was observed for other causes: spine injury improved 3 points early, 2 points late; head injury 2 points early, 1 point late; and ALS 2 points early, 1 point late. The 4 patients with spinocerebellar degenerative disease maintained a 2-point improvement on the Modified Ashworth Scale from early to late follow-up. 


\section{Selective dorsal rhizotomy for non-cerebral palsy spasticity}

Long-term efficacy was best in the $\mathrm{CP}$ patients, who were the youngest on average at surgery (12 years, compared with 42 years for MS, 28 years for trauma, and 51 years for ALS/spinocerebellar degeneration) and maintained a 3-point improvement on the Modified Ashworth Scale on late follow-up.

Other outcome measures were assessed as well, although not segregated by origin of spasticity. Lower limb spasticity, range of motion in affected joints, gait, bladder function, upper limb spasticity, and speech and cognition were all evaluated at early and late follow-up. All measures showed improvement, which did not appear to dissipate over time. All of the patients (100\%) showed improved lower limb spasticity at early and late follow-up. There were no cases of worsening of any outcome variable in any patient at late follow-up, and $20 \%$ of patients who were nonambulatory preoperatively were able to walk at early and late follow-up.

The only other series with a comparable number of diverse patients described spasticity from MS in 6 patients, and 1 patient each with spinal cord injury, nonspecific myelopathy, and cerebral hemorrhage. ${ }^{17}$ Functional motility, pinprick sensation, and bladder function were all assessed preoperatively and 6-26 months postoperatively. Among the 6 patients with MS, all were paraplegic and wheelchair-bound preoperatively. Two patients became ambulatory postoperatively with assistive devices. One patient experienced 4 months of relief from deafferentation pain postoperatively, and another experienced lasting relief from spasticity-associated leg pain. There was 1 instance of transient deterioration in bladder function; otherwise, no adverse effects were reported. Spasticity was described as abolished in 4 patients, markedly reduced in 1 patient, and slightly reduced in 1 patient.

The patient with a spinal injury in this study underwent unilateral SDR for leg paresis and spasticity with deafferentation pain. At 20 months postoperatively, spasticity was abolished, ambulation had improved significantly, and pain was transiently relieved for 3 months. There was no change in normal bladder function. The patient with a diagnosis of nonspecific myelopathy was described as showing improvement in ambulation, and marked reduction in unilateral lower-extremity spasticity and associated pain at 12 months postoperatively. Normal bladder function and pinprick sensation were unchanged. The patient with cerebral hemorrhage was the only one to undergo cervical SDR, which was reported to markedly reduce spasticity in the affected arm with some return of functional motility. There was a possible slight deterioration in pinprick sensation.

A single-institution study that reviewed results of SDR on pediatric patients with spastic hemiparesis included 13 patients who underwent surgery between 2006 and 2008, 9 of whom had a diagnosis of CP. ${ }^{23}$ Of the remaining patients, 2 had a history of traumatic brain injury, 1 had a history of brain tumor, and 1 had an intracerebral hemorrhage from an arteriovenous malformation. All patients were reported to have demonstrated a decrease in resting muscle tone following SDR. Detailed outcome results were specified for the brain tumor patient, in whom stride length on the hemiparetic side improved from 25.5 inches preoperatively to 40 inches postoperatively, and time to walk 30 feet improved from 12.62 to $5.43 \mathrm{sec}$ onds. Overall results in this study were characterized as consistent with previously published results of SDR for spastic diplegia in randomized controlled trials. ${ }^{20,21,34,37}$

\section{Spasticity From Spinal Cord Injury}

In addition to the previously described reports including brain and spinal cord injured patients, ${ }^{17,28}$ an early report from 1976 described the results of SDR in 4 patients with posttraumatic paraplegia as well as 5 patients with $\mathrm{CP} .{ }^{26}$ An additional patient with postmeningitis progressive spastic paraplegia was categorized with the trauma patients. The patients with $\mathrm{CP}$ underwent surgery at between 8 and 10 years of age; the 5 trauma patients underwent surgery between 17 and 23 years of age. Given the difference in ages and patterns of spasticity described in the 2 groups, direct comparison of outcomes is problematic. Outcomes in this trauma group appear similar to those from the posttraumatic patients in the previously described studies. ${ }^{17,28}$

The postmeningitis patient in the present report ${ }^{26} \mathrm{had}$ severely debilitating flexion spasm in the hips; postoperatively, the patient experienced significantly improved sitting ability. Of the remaining patients, 3 were ambulatory with assistive devices preoperatively, and all experienced significantly improved gait postoperatively with 2 able to walk without help. The last patient had a complete midthoracic spinal cord level and severe lower-extremity flexion spasm interfering with sitting. Selective dorsal rhizotony permitted ventral posture, abduction, and sitting posture in this patient. The authors clarified that attempts were made to purposefully preserve some degree of useful spasticity, particularly in quadriceps femoris and gluteus muscles, to assist with walking and upright posture.

An additional case report was published in 2002 describing a 26-year-old man with a diagnosis of systemic lupus erythematosus who subsequently developed transverse myelitis. ${ }^{38}$ The patient experienced significant progressive spastic paraplegia, dysthetic pain, and impaired bladder control, rendering him bedridden and dependent in activities of daily living. The patient underwent SDR 1 year after onset of transverse myelitis. He was described as completely independent in ambulation and self-care at 10 months postoperatively, without adverse effects from the surgery.

\section{Spasticity From Stroke}

A report of 2 patients who developed severe unilateral lower-extremity spasticity following ischemic stroke described good results from unilateral SDR..$^{14}$ Both patients had very poor tolerance of oral muscle relaxants. The first patient was 68 years old at the time of an acute, distal, left anterior cerebral artery infarct that resulted in progressive, severe, and painful right leg spasticity over the next 6 months. The patient could stand with a cane but not walk. A unilateral right-sided SDR restored the patient's ability to walk with a cane and significantly improved activities of daily living for more than 1 year after surgery.

The patient in the second report suffered a right-sided putamenal stroke at 89 years of age that resulted in severe 
left hemiparesis and spasticity, causing intolerable pain in the leg that was particularly acute when the patient was moved by others (during transfers). Unilateral left SDR resulted in complete relief of spasticity-associated pain, with muscle tone in the leg evaluated as normal to hypotonic.

\section{Spasticity From Neurodegenerative Disease}

In addition to the previously described 3 patients with ALS and 4 with spinocerebellar degeneration, ${ }^{28}$ there has also been another report of SDR used in 2 cases of spasticity secondary to neurodegenerative disease. ${ }^{16}$ At 18 years old, the first patient was given a diagnosis of hypomyelination with atrophy of the basal ganglia and cerebellum, having developed severe progressive spastic quadriplegia and dysarthria between ages 2 and 12 years. At age 19 years, the patient underwent placement of an intrathecal baclofen pump, which was removed 2 weeks later due to infection, although caregiver satisfaction with this treatment modality was described as minimal. Selective dorsal rhizotomy was subsequently performed, which resulted in the disappearance of leg spasticity. The postoperative course was complicated by a urinary tract infection, recurrent migrating arthritis, and ventral dislocation of the L-1 spinous process requiring surgical revision. Over the next 3 years, the clinical course remained progressive, although spasticity never returned in the legs.

A second patient with an uncharacterized mitochondrial genetic disorder and progressive spastic quadriparesis underwent SDR at age 8 years of age with disappearance of leg spasticity. Over the following 3 years, the legs remained stable with oral baclofen treatment, but the disease process progressed; spasticity in the arms increased, leading to disabling contractures. Extension posturing of the trunk (arching of the back from severe muscle spasm) eventually interfered with sitting.

\section{Spasticity From Congenital Brain Malformation}

A relatively recent study described SDR in the setting of disorders of neuronal migration. The report included only 2 patients, but both had spastic diplegia secondary to congenital brain malformations..$^{35}$ These patients represented $1.77 \%$ of the 113 SDR cases performed over 15 years at 1 institution, and were the only patients without a diagnosis of CP. The first patient had a diagnosis of semilobar holoprosencephaly and underwent surgery at 3.5 years of age. Preoperatively, this patient was ambulatory. Over the reported 5-year follow-up period, significant improvements were sustained in ambulation and lowerextremity range of motion.

The second patient had open-lip schizencephaly and left frontal cortical dysplasia that led to a spastic triplegia involving the left arm and both legs. Surgery was performed at age 6 years. In this patient, who was also ambulatory preoperatively, significant improvement in gait and lower-extremity muscle tone was observed 2 years postoperatively.

\section{Spasticity From MS}

Previously described studies included a total of 59 patients with spasticity from MS, among patients with other diagnoses. ${ }^{17,28,29}$ An additional study focused specifically on the results of SDR in patients with spasticity from this cause. ${ }^{32}$ This series was composed of 15 patients with a diagnosis of MS who underwent SDR between 1974 and 1981. Thirteen of the patients were women, the duration between diagnosis of MS and surgery ranged from 6 to 30 years (average 12 years), and age at surgery ranged from 31 to 58 years old. Postoperative follow-up in this cohort ranged from 1 to 8 years.

Preoperative functional status in the patients was described as completely bedridden in 11 patients and wheelchair dependent in the other 4 patients. Postoperatively, 8 patients were able to walk with crutches, 1 was able to stand upright, 3 were wheelchair dependent, and only 2 remained bedridden. Twelve of the patients demonstrated significant improvement in spontaneous flexion postures. However, 6 patients did undergo further orthopedic surgeries for contractions within 2 months of their SDR. Two patients exhibited deterioration of motor function, described as corresponding to 1 spinal level. It was also noted that SDR partially reduced sensation in 1 or more spinal levels in 13 patients, although this was described as having no practical consequences. The conclusion from this report was that 12 of 15 patients experienced satisfactory symptomatic improvement following SDR.

\section{Spasticity From Hereditary Spastic Paraparesis}

Hereditary spastic paraparesis refers to a family of genetically and clinically diverse disorders that have progressive lower-extremity spasticity and weakness in common. These disorders are generally characterized by mode of inheritance and symptoms. At least 52 different mutations have been associated with this disease process, which can be transmitted in an autosomal dominant, autosomal recessive, or X-linked fashion. ${ }^{10}$ Hereditary spastic paraparesis is characterized as uncomplicated if the spasticity is associated only with mild lower-extremity loss of proprioception and sphincter dysfunction, or complex if it associated with any other neurological problems. ${ }^{31}$

Only 1 report was identified that included a patient with hereditary spastic paraparesis who underwent SDR. ${ }^{5}$ This study examined whether adherence to strict patient selection criteria for SDR resulted in improved outcomes, although their criteria explicitly included hereditary spastic paraparesis as an appropriate indication for surgery. Of 53 children referred to the authors for possible SDR, only 19 were determined to be suitable surgical candidates. The SDR cohort included 1 of 4 patients initially evaluated who had a diagnosis of hereditary spastic paraparesis; specific rationales for excluding the other 3 patients were not given. Detailed clinical evaluations were performed both preoperatively and an average of 18 months postoperatively. Although individual patient data were not reported, collective results showed statistically significant improvement in a large number of measurements. Walking speed, step length, hip and knee extensor power, ankle dorsiflexion range and power, and ankle plantar flexion range were among the parameters that improved. Patients with hereditary spastic paraparesis may benefit from further detailed analysis of potential benefits from SDR. 


\section{Selective dorsal rhizotomy for non-cerebral palsy spasticity}

\section{Discussion}

This study was motivated in part by a 2-year-old patient referred to our neurosurgery program for possible unilateral SDR for fixed, distal, left lower limb spasticity. The patient had extensive polymicrogyria of the right perisylvian region and no other historical, clinical, or radiographic findings suggestive of $\mathrm{CP}$. This patient was ambulatory with an orthosis, with no other assistive device necessary. As of the most recent evaluation, the family did not wish to pursue neurosurgical intervention.

A meta-analysis of SDR trials that randomized CP patients with spastic diplegia to surgery in addition to physical therapy, or physical therapy alone, concluded that the surgical arm exhibited superior results after 1 year in a statistically significant fashion. ${ }^{20}$ Outcome measures that showed improvement included the Ashworth scale for spasticity and the Gross Motor Function Measure for motor function. A direct relationship between percentage of dorsal root fascicles transected and functional improvement was observed. Subsequent large nonrandomized studies have found similar functional improvements that persist at up to 26 years postoperatively., $4,18,36$

The presumed mechanism by which SDR alleviates spasticity is by modulation of afferent inputs to lower motor neurons to compensate for a loss of upper motor neuron regulation, although the details of this mechanism are poorly understood. ${ }^{15}$ The most common imaging finding associated with $\mathrm{CP}$ is periventricular leukomalacia, which is associated with necrosis of the periventricular and subcortical white matter. This region is the most vulnerable to hypoperfusion during hypotension, and may suffer reperfusion injury from oxygen free radicals and excitatory amino acids. It has been speculated that periventricular leukomalacia-associated injury to the projection fibers from the primary motor cortex is the underlying basis for spastic $\mathrm{CP}$. The topographic arrangement of these projection fibers would lead leg fibers, traversing closer to the ventricle, to be injured preferentially over arm fibers, depending on the extent of the periventricular leukomalacia. ${ }^{12,13}$

Muscle tone is determined largely by alpha motor neuron output in the ventral horn of the spinal cord; excitatory and inhibitory modulation may be disrupted by periventricular leukomalacia-injured descending motor fibers. ${ }^{12,13}$ Spasticity is generally characterized as the result of abnormal feedback loops between dorsal and ventral nerves. Ventral rhizotomy does not yield acceptable results. ${ }^{1}$ This model implies that success of SDR should be independent of specific features of the upper motor neuron disease process, as is reflected to some degree in its broad efficacy across the heterogeneous CP population. The data reviewed in this report suggest that SDR may be appropriate for an even wider range of upper motor neuron disease processes and may even extend to upper motor neuron pathologies that are extracranial, progressive, or both.

Our literature review identified many reports of patients undergoing SDR for diagnoses other than CP, although only 4 pathologies were represented in more than 2 cases: MS, spinal cord injury, neurodegenerative dis- ease, and traumatic brain injury. The 74 patients with MS comprised the largest cohort by diagnosis, across 1 study specific to this disease process ${ }^{32}$ and 3 studies that included multiple diagnoses..$^{17,28,29}$ Only 1 of the latter studies ${ }^{28}$ specified diagnosis-specific outcomes; the 52 patients with MS in that study showed a mean improvement of 2 points on the Ashworth scale at more than 1 year after surgery. The MS-specific series, although describing 12 of 15 outcomes as satisfactory, did not use Ashworth scoring as part of the patient evaluation. Similar heterogeneity in patient evaluation and outcome reporting was found in the spinal cord injury, ${ }^{17,26,28}$ traumatic brain injury, ${ }^{28,29}$ and neurodegenerative disease ${ }^{16,28}$ cohorts. Although the MS data are promising, there is insufficient evidence to consider any of these diagnoses to be indications for SDR.

Limitations to the available data are clear. While certainly justifying further investigation, there is an inadequate empirical basis for widespread expansion of SDR outside of established diagnostic criteria. Studies have suggested that SDR may be inappropriate in some patients, such as those more than 10 years of age, ${ }^{19}$ and some authors continue to question its efficacy even within the most thoroughly studied populations. ${ }^{6}$ It is also the case that the full complexity of spinal motor circuits is not completely understood, nor is the role and mechanism of upper motor neuron and sensory input into these circuits..$^{15}$ It is therefore our conclusion that SDR, as an irreversible neuroablative procedure, should continue to be performed only with great caution.

\section{Conclusions}

Selective dorsal rhizotomy is a well-studied and effective therapy for lower-extremity spasticity resulting from $\mathrm{CP}$ and may be beneficial in other types of upper motor neuron disease. This procedure has not been systematically studied in contexts outside of CP. Multiple sclerosis and traumatic spinal cord injury are the most commonly reported non-CP diagnoses in patients who have undergone SDR. Patients with traumatic brain injury, near drowning, progressive neurological disease, spastic hemiplegia, and age greater than 45 years have undergone SDR despite falling outside common inclusion criteria for surgery. Although reported results are described as satisfactory, no standardized outcomes data are available outside of the CP population. Limited information from case reports is suggestive that patients with a variety of diagnoses may derive some benefit from this procedure at least in the short to intermediate term. Consideration should be given to prospective multiinstitutional enrollment of patients in a standardized outcomes database.

\section{Disclosure}

The authors report no conflict of interest concerning the materials or methods used in this study or the findings specified in this paper.

Author contributions to the study and manuscript preparation include the following. Conception and design: Gump. Acquisition of data: Gump. Analysis and interpretation of data: Gump. Drafting the article: Gump, Mutchnick. Critically revising the article: all authors. Reviewed submitted version of manuscript: Gump, Mutchnick. Approved the final version of the manuscript on behalf of all authors: Gump. 


\section{References}

1. Albright AL: Spasticity and movement disorders, in Albright AL, Pollack IF, Adelson PD (eds): Principles and Practice of Pediatric Neurosurgery, ed 2. New York: Thieme, 2008, pp 1121-1138

2. Ashwal S, Russman BS, Blasco PA, Miller G, Sandler A, Shevell M, et al: Practice parameter: diagnostic assessment of the child with cerebral palsy: report of the Quality Standards Subcommittee of the American Academy of Neurology and the Practice Committee of the Child Neurology Society. Neurology 62:851-863, 2004

3. Barolat G: Dorsal selective rhizotomy through a limited exposure of the cauda equina at L-1. Technical note. J Neurosurg 75:804-807, 1991

4. Bolster EA, van Schie PE, Becher JG, van Ouwerkerk WJ, Strijers RL, Vermeulen RJ: Long-term effect of selective dorsal rhizotomy on gross motor function in ambulant children with spastic bilateral cerebral palsy, compared with reference centiles. Dev Med Child Neurol 55:610-616, 2013

5. Cole GF, Farmer SE, Roberts A, Stewart C, Patrick JH: Selective dorsal rhizotomy for children with cerebral palsy: the Oswestry experience. Arch Dis Child 92:781-785, 2007

6. Crilly MA: Selective dorsal rhizotomy remains experimental in cerebral palsy. BMJ 345:e6670-e6679, 2012 (Letter)

7. Dudley RW, Parolin M, Gagnon B, Saluja R, Yap R, Montpetit K, et al: Long-term functional benefits of selective dorsal rhizotomy for spastic cerebral palsy. Clinical article. J Neurosurg Pediatr 12:142-150, 2013

8. Farmer JP, Sabbagh AJ: Selective dorsal rhizotomies in the treatment of spasticity related to cerebral palsy. Childs Nerv Syst 23:991-1002, 2007

9. Fasano VA, Broggi G, Barolat-Romana G, Sguazzi A: Surgical treatment of spasticity in cerebral palsy. Childs Brain 4: 289-305, 1978

10. Finsterer J, Löscher W, Quasthoff S, Wanschitz J, Auer-Grumbach M, Stevanin G: Hereditary spastic paraplegias with autosomal dominant, recessive, X-linked, or maternal trait of inheritance. J Neurol Sci 318:1-18, 2012

11. Foerster O: On the indications and results of the excision of posterior spinal nerve roots in men. Surg Gynecol Obstet 16:463-474, 1913

12. Folkerth RD: Neuropathologic substrate of cerebral palsy. J Child Neurol 20:940-949, 2005

13. Folkerth RD: Periventricular leukomalacia: overview and recent findings. Pediatr Dev Pathol 9:3-13, 2006

14. Fukuhara T, Kamata I: Selective posterior rhizotomy for painful spasticity in the lower limbs of hemiplegic patients after stroke: report of two cases. Neurosurgery 54:1268-1273, 2004

15. Garcia-Campmany L, Stam FJ, Goulding M: From circuits to behaviour: motor networks in vertebrates. Curr Opin Neurobiol 20:116-125, 2010

16. Grunt S, van der Knaap MS, van Ouwerkerk WJ, Strijers RL, Becher JG, Vermeulen RJ: Effectiveness of selective dorsal rhizotomy in 2 patients with progressive spasticity due to neurodegenerative disease. J Child Neurol 23:818-822, 2008

17. Laitinen LV, Nilsson S, Fugl-Meyer AR: Selective posterior rhizotomy for treatment of spasticity. J Neurosurg 58:895899,1983

18. Langerak NG, Tam N, Vaughan CL, Fieggen AG, Schwartz MH: Gait status 17-26 years after selective dorsal rhizotomy. Gait Posture 35:244-249, 2012

19. MacWilliams BA, Johnson BA, Shuckra AL, D'Astous JL: Functional decline in children undergoing selective dorsal rhizotomy after age 10. Dev Med Child Neurol 53:717-723, 2011

20. McLaughlin J, Bjornson K, Temkin N, Steinbok P, Wright V, Reiner A, et al: Selective dorsal rhizotomy: meta-analysis of three randomized controlled trials. Dev Med Child Neurol 44:17-25, 2002

21. McLaughlin JF, Bjornson KF, Astley SJ, Graubert C, Hays
RM, Roberts TS, et al: Selective dorsal rhizotomy: efficacy and safety in an investigator-masked randomized clinical trial. Dev Med Child Neurol 40:220-232, 1998

22. Munro D: Anterior-rootlet rhizotomy; a method of controlling spasm with retention of voluntary motion. N Engl J Med 246:161-166, 1952

23. Oki A, Oberg W, Siebert B, Plante D, Walker ML, Gooch JL: Selective dorsal rhizotomy in children with spastic hemiparesis. Clinical article. J Neurosurg Pediatr 6:353-358, 2010

24. Park TS: Selective dorsal rhizotomy: an excellent therapeutic option for spastic cerebral palsy. Clin Neurosurg 47:422-439, 2000

25. Peacock WJ, Arens LJ, Berman B: Cerebral palsy spasticity. Selective posterior rhizotomy. Pediatr Neurosci 13:61-66, 1987

26. Privat JM, Benezech J, Frerebeau P, Gros C: Sectorial posterior rhizotomy, a new technique of surgical treatment for spasticity. Acta Neurochir (Wien) 35:181-195, 1976

27. Sacco DJ, Tylkowski CM, Warf BC: Nonselective partial dorsal rhizotomy: a clinical experience with 1-year follow-up. Pediatr Neurosurg 32:114-118, 2000

28. Salame K, Ouaknine GER, Rochkind S, Constantini S, Razon N: Surgical treatment of spasticity by selective posterior rhizotomy: 30 years experience. Isr Med Assoc J 5:543-546, 2003

29. Schijman E, Erro MG, Meana NV: Selective posterior rhizotomy: experience of 30 cases. Childs Nerv Syst 9:474-477, 1993

30. Sherrington CS: Decerebrate rigidity, and reflex coordination of movements. J Physiol 22:319-332, 1898

31. Siddique N, Sufit R, Siddique T: Degenerative motor, sensory, and autonomic disorders, in Goetz CG (ed): Textbook of Clinical Neurology, ed 2. Philadelphia: Saunders, 2003, pp 759-785

32. Sindou M, Millet MF, Mortamais J, Eyssette M: Results of selective posterior rhizotomy in the treatment of painful and spastic paraplegia secondary to multiple sclerosis. Appl Neurophysiol 45:335-340, 1982

33. Steinbok P: Selective dorsal rhizotomy for spastic cerebral palsy: a review. Childs Nerv Syst 23:981-990, 2007

34. Steinbok P, Reiner AM, Beauchamp R, Armstrong RW, Cochrane DD, Kestle J: A randomized clinical trial to compare selective posterior rhizotomy plus physiotherapy with physiotherapy alone in children with spastic diplegic cerebral palsy. Dev Med Child Neurol 39:178-184, 1997

35. Tubbs RS, Bui CJ, Loukas M, Shoja MM, Oakes WJ: Partial dorsal rhizotomy for spasticity in children with congenital brain malformations. Report of two cases. J Neurosurg 106 (5 Suppl):407-409, 2007

36. van Schie PE, Schothorst M, Dallmeijer AJ, Vermeulen RJ, van Ouwerkerk WJ, Strijers RL, et al: Short- and long-term effects of selective dorsal rhizotomy on gross motor function in ambulatory children with spastic diplegia. Clinical article. J Neurosurg Pediatr 7:557-562, 2011

37. Wright FV, Sheil EM, Drake JM, Wedge JH, Naumann S: Evaluation of selective dorsal rhizotomy for the reduction of spasticity in cerebral palsy: a randomized controlled trial. Dev Med Child Neurol 40:239-247, 1998

38. Yang TF, Lee SS, Lin PH, Chen H, Chan RC: Effect of selective posterior rhizotomy on transverse myelitis in a patient with systemic lupus erythematosus. Am J Phys Med Rehabil 81:467-468, 2002

Manuscript submitted July 13, 2013.

Accepted August 16, 2013.

Please include this information when citing this paper: DOI: 10.3171/2013.8.FOCUS13294.

Address correspondence to: William C. Gump, M.D., Norton Neuroscience Institute, Division of Pediatric Neurosurgery, 210 E. Gray St., Ste. 1102, Louisville, KY 40202. email: william.gump@ nortonhealthcare.org. 